[16] S. J. Gardiner, Positive harmonic majorization of the real part of a holomorphic function, Proc. Amer. Math. Soc. (to appear).

[17] S. J. Gardiner, Sets of determination for harmonic functions, Trans. Amer. Math. Soc. (to appear).

[18] S. J. Gardiner, Angular limits of holomorphic functions which satisfy an integrability condition, Mh. Math. (to appear).

[19] W. K. Hayman and T. J. Lyons, Bases for positive continuous functions, J. London Math. Soc. (2) 42 (1990), 292-308.

[20] L. L. Helms, Introduction to potential theory. Krieger: New York, 1975.

[21] C. Pommerenke, Univalent functions. Vandenhoeck and Ruprecht: Göttingen, 1975.

[22] B. Rodin and S. Warschawski, Rernarks on a paper of K. Burdzy, J. Analyse Math. 46 (1980), 251-60.

[23] S. Sastry, An extremal length proof of Burdzy's theorem (preprint).

Stephen J. Gardiner Department of Mathematics and Statistics

McGill University

Montréal

Québec

Canada H3A 2K6
Permanent address:

Department of Mathematics

University College Dublin 4

Ireland

\section{INTERNAL FORCING AXIOMS: MARTIN'S AXIOM AND THE PROPER FORCING AXIOM}

Dedicated to the memory of Alan H. Mekler.

\section{Eoin Coleman}

In the course of the last twenty-five years research in the combinatorics of partially ordered sets has resulted in the discovery of new set-theoretic hypotheses - sometimes dubbed internal forcing axioms. This elementary article presents in section 1 the simplest of these (Martin's Axiom). In section 2 we look at some applications (the completeness of the category ideal, Lusin sets, $Q$-sets, problems of Moore, Alexandroff, Suslin, Whitehead and Kaplansky). Finally in section 3 we deal briefly with the Proper Forcing Axiom, a powerful generalization of Martin's Axiom. We've collected the relevant references in an annotated bibliography in section 4, rather than in the body of the text.

We try to show concretely how internal forcing axioms work (giving complete proofs whenever feasible), stressing the resemblance to the classical diagonal arguments of Baire and Cantor. In our choice of applications we seek to underline the fact that mathematical conjectures having no apparent set-theoretic reference may depend for their resolution on axioms beyond those of ordinary set theory. To put it another way, there are at least three truth values in mathematics: true, false, and independent of ordinary set theory.

\section{Section 1: Forcing}

Internal forcing axioms are about forcings. Let us recall that a forcing is simply a partial order, i.e. a pair $\mathbb{P}=(P, \leq)$ such that $P$ is a non-empty set, $\leq$ is a reflexive antisymmetric transitive 
binary relation on $P$ (so, for all $p, q, r \in P$, (i) $p \leq p$, (ii) if $p \leq q$ and $q \leq p$, then $p=q$, and (iii) if $p \leq q$ and $q \leq r$, then $p \leq r)$. Elements of $P$ are called conditions. Conditions $p$ and $q$ are compatible iff they have a common upper bound in $P$, i.e. $(\exists r \in P)(p \leq r$ and $q \leq r)$; otherwise $p$ and $q$ are incompatible. A subset $D \subseteq P$ is dense in $\mathbb{P}$ iff $(\forall p \in P)(\exists r \in D)(p \leq r)$. A nonempty subset $G$ of $P$ is a flter in $\mathbb{P}$ iff $(\forall p, q \in G)(\exists r \in G)(p \leq r$ and $q \leq r)$ and $(\forall p \in P)(\forall q \in G)$ (if $p \leq q$, then $p \in G$ ). Finally if $\mathcal{D}$ is a family of dense sets in $\mathbb{P}$, we say that a filter $G$ in $\mathbb{P}$ is $\mathcal{D}$-generic iff for every $D \in \mathcal{D}, G \cap D \neq \emptyset$.

To sort out these definitions, consider the following situation.

Example 1.1: Adding a Cohen real. Let $P$ be the set $\{f: f$ is a function from a finite subset of $\mathbb{N}$ to $\{0,1\}\}$ and define a partial ordering on $P$ by $f \leq g$ iff $g$ extends $f$, i.e. dom $f \subseteq \operatorname{dom} g$ and $g \mid \operatorname{dom} f=f$. Certainly $\mathbb{P}=(P, \leq)$ is a forcing. Conditions $f$ and $g$ are compatible iff they agree on $\operatorname{dom} f \cap \operatorname{dom} g$, in which case the union $f \cup g$ is a condition extending $f$ and $g$. So if $G$ is a filter in $\mathbb{P}$, then $\bigcup G=\bigcup\{f: f \in G\}$ is a function from a subset of $N$ to $\{0,1\}$, since the union of compatible functions is itself a function. Note also that if $f \in G$ and $n \in \operatorname{dom} f$, then $(\bigcup G)(n)=f(n)$. Examples of dense sets are the sets $C_{m}=\{g \in P: m \in \operatorname{dom} g\}$ for each $m \in \mathbb{N}$ : given any $f \in P$, either $f \in C_{m}$, or $m \notin \operatorname{dom} f$ and then $g=f \cup(m, 0)$ belongs to $C_{m}$ and $f \leq g$. Observe that the dense sets which $G$ intersects determine to some extent the function $\bigcup G$ : for example, if $G \cap C_{m} \neq \emptyset$, then $m \in \operatorname{dom} \bigcup G$. So if $G$ is $\mathcal{C}$-generic where $\mathcal{C}=\left\{C_{m}: m \in \mathbb{N}\right\}$, then $\bigcup G$ is a function from (all of) $\mathbb{N}$ to $\{0,1\}$. If $\mathcal{D} \supseteq \mathcal{C}$ and $G$ is $\mathcal{D}$-generic, then $\bigcup G$ is called a Cohen real. Note that a Cohen real does not belong to $P$, since its domain is the infinite set $\mathbb{N}$.

Internal forcing axioms are putatively consistent answers to the natural question: for which forcings $P$ and families $\mathcal{D}$ of dense sets in $P$ does there exist a $\mathcal{D}$-generic filter $G$ in $\mathbb{P}$ ? The first and weakest internal forcing axiom is a very easy Cantorian diagonal argument.

Proposition 1.2. If $\mathbb{P}$ is a forcing and $\mathcal{D}$ is a countable family of dense sets in $\mathbb{P}$, then there is a $\mathcal{D}$-generic filter $G$ in $\mathbf{P}$.
Proof: Enumerate $\mathcal{D}$ as $\left\{D_{n}: n \in \mathbb{N}\right\}$ and by induction on $n$ choose $p_{n}$ such that $p_{0} \in P$ and for $n \geq 1, p_{n} \in D_{n-1}$ and $p_{n-1} \leq p_{n}$ (possible since $D_{n-1}$ is dense in $\mathbb{P}$ ). Now let $G=\{q \in$ $\left.P:(\exists n \in \mathbb{N})\left(q \leq p_{n}\right)\right\}$.

We'll apply this to prove a very well-known theorem.

Corollary 1.3 (The Baire category theorem). If $X$ is a compact Hausdorff space (or a complete metric space) and $A_{n}$ is a (topologically) dense open subset of $X$ for $n \in \mathbb{N}$, then $\bigcap\left\{A_{n}: n \in \mathbb{N}\right\}$ is non-empty.

Proof: Let $P$ be the set $\{p \subseteq X: p$ is a non-empty open set $\}$ and define $p \leq q$ iff $q \subseteq p$. For $n \in \mathbb{N}$ the set $D_{n}=\{p \in P: \mathrm{Cl}(p) \subseteq$ $\left.A_{n}\right\}$ is dense in $\mathbb{P}$ : given $q$ in $P$, we know $A_{n} \cap q \neq \emptyset$, so since $X$ is regular there is $p \in P$ such that $\mathrm{Cl}(p) \subseteq A_{n} \cap q$; now $p \in D_{n}$ and $q \leq p$. Proposition 1.2 yields a filter $G$ which intersects each $D_{n}$ non-trivially. Let $A$ be $\bigcap\{\mathrm{Cl}(p): p \in G\}$. Clearly $A \subseteq \bigcap\left\{A_{n}: n \in \mathbb{N}\right\}$ since $G \cap D_{n} \neq \emptyset$. Note also that for each finite $F \subseteq G, \bigcap\{\mathrm{Cl}(p): p \in F\}$ is non-empty: $G$ is a filter, so there is $r \in G(\forall p \in F)(p \leq r)$ and so $\emptyset \neq r \subseteq \bigcap\{\mathrm{Cl}(p): p \in F\}$. Now since $X$ is compact, it follows that $A$ is non-empty.

As it stands, Proposition 1.2 is the best one can do. If $\mathcal{D}$ is uncountable, the conclusion does not necessarily hold.

Proposition 1.4. There is a forcing $Q$ and an uncountable family $\mathcal{R}$ of dense sets for which there is no generic $\mathcal{R}$-filter.

Proof: Let $I$ be an uncountable set, let $Q$ be $\{f: f$ is a function from a finite subset of $\mathbb{N}$ to $I\}$, and define $f \leq g$ iff $g$ extends $f$. For $i \in I$, the set $R_{i}=\{f \in Q: i \in$ range $f\}$ is dense in $Q$. Taking $\mathcal{R}=\left\{R_{i}: i \in I\right\}$, we note that if $G$ were an $\mathcal{R}$-generic filter, then $\bigcup G$ would be a function from a (countable) subset of $\mathrm{N}$ onto the uncountable set $I-$ an impossibility.

We can make precise an important difference between situations 1.1 and 1.4 by considering the sizes of the sets of pairwise incompatible conditions in the respective forcings. 
Definition 1.5. Suppose $P$ is a forcing.

(1) An antichain in $\mathbb{P}$ is a set $A \subseteq P$ of pairwise incompatible conditions.

(2) We say that $\mathbb{P}$ has the countable chain condition ( $\mathbb{P}$ is c.c.c.) iff every antichain in $\mathbb{P}$ is countable.

(3) A subset $C$ of $P$ is a chain in $\mathrm{P}$ iff $(\forall p, q \in C)(p \leq q$ or $q \leq$ $p)$.

Some authors refer to (2) as the countable antichain condition.

Thus the Cohen forcing $P$ of 1.1 is c.c.c. trivially, since $P$ is itself a countable set, whereas in $1.4 Q$ is not c.c.c., since $A=\left\{f_{i}: i \in I\right\}$ is an uncountable antichain, where $f_{i}(0)=i$ for $i \in I$. By restricting attention to c.c.c. forcings, we avoid the counterexample of 1.4 at least, and it makes sense to reformulate Proposition 1.2 for c.c.c. forcings and uncountable families of dense sets.

Definition 1.6. We let $\mathrm{MA}_{\kappa}$ abbreviate the hypothesis: if $P$ is a c.c.c. forcing, $\mathcal{D}$ is a family of dense sets in $\mathbf{P}$ and $\mathcal{D}$ has cardinality at most $\kappa$, then there is a $\mathcal{D}$-generic filter $G$ in $\mathbf{P}$.

Just to clear up some notation: we use $\kappa, \lambda, \ldots$ to denote infinite cardinals; the first infinite cardinal is $\aleph_{0}$; the first uncountable cardinal is $\aleph_{1}$. For a set $X,|X|$ is the cardinality of $X$ $P(X)$ is the power set of $X$. The cardinal $2^{|X|}$ is $\mid\{f: f$ is a function from $X$ to $\{0,1\}\} \mid ; \lambda^{+}$is the least cardinal greater than $\lambda$ For example, $\aleph_{1}=\aleph_{0}^{+}, \aleph_{0}=|\mathbb{N}|, 2^{\aleph_{0}}=|\mathbb{R}|$, and $2^{|X|}=|P(X)|$ (identifying subsets of $X$ with their characteristic functions).

For each infinite cardinal $\kappa$ we obtain a version of 1.2 for c.c.c. forcings and families of dense subsets of cardinality at most $\kappa$. Some are obviously true; some are false.

Proposition 1.7. (1) $\mathrm{MA}_{\aleph_{0}}$ is true. (2) $\mathrm{MA}_{\kappa}$ implies $\kappa<2^{\aleph_{0}}$. (3) $\mathrm{MA}_{\lambda}$ is false for every $\lambda \geq 2^{\aleph_{0}}$.

Proof: Proposition 1.2 clearly implies 1.7 (1). Part (3) follows from (2). For (2), we show that there is no mapping $F$ from $\kappa$ onto the set ${ }^{N} 2=\{f: f$ is a function from $N$ to $\{0,1\}\}$. Suppose that $F$ maps $\kappa$ to ${ }^{\mathrm{N}} 2$. Let $H=$ range $F$. For each $h \in H$, let $R_{h}=\{f \in P:(\exists n \in \operatorname{dom} f)(f(n)=1-h(n))\}$, where $\mathbf{P}$ is the
Cohen forcing of Example 1.1. Note that $R_{h}$ is dense in $\mathbf{P}$. As in 1.1 , let $C_{m}=\{f \in P: m \in \operatorname{dom} f\}$. Now $\mathcal{D}=\left\{C_{m}, R_{h}: m \in\right.$ $\mathbf{N}, h \in H\}$ is a family of dense sets in $\mathbf{P}$ and $\mathcal{D}$ has cardinality at most $\kappa$ (recall $\kappa+\aleph_{0}=\kappa$ since $\kappa$ is infinite). By MA $\mathrm{A}_{\kappa}$ there is a $\mathcal{D}$-generic filter $G$ in $\mathbb{P}$. The Cohen real $\bigcup G$ belongs to ${ }^{\mathrm{N}_{2}}$ (since $G \cap C_{m} \neq \emptyset \forall m \in \mathbb{N}$ ), but does not belong to $H$ (since fог each $h \in H, G \cap R_{h} \neq \emptyset$, so $(\exists n \in \mathbb{N})(\bigcup G(n)=1-h(n))$, giving $\bigcup G \neq h)$. Thus $F$ is not onto.

Remark that letting $\kappa=\aleph_{0}$ in (2) and using (1), one obtains Cantor's theorem: $2^{\aleph_{0}}>\aleph_{0}$. The original diagonal argument runs as follows: if $\left\{h_{n}: n \in \mathbb{N}\right\} \subseteq \mathcal{N}_{2}$, then the function $g$ defined by $g(n)=1-h_{n}(n)$ for $n \in \mathbb{N}$ belongs to $\mathbb{N}_{2}$ but differs in the $n$th place from each $h_{n}$. In the argument from 1.7 (1) (2), one finds the required function $g$ by considering the c.c.c. forcing consisting of the finite approximations to $g$ and defining appropriate dense sets.

Guided by the information in Proposition 1.7 we write down Martin's Axiom.

Definition 1.8. Martin's Axiom MA is the hypothesis $(\forall \kappa<$

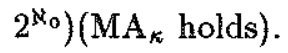
ately:

From the definition and Proposition 1.7 we obtain immedi-

Corollary 1.9. (1) The Continuum Hypothesis CH $\left(2^{\aleph_{0}}=\aleph_{1}\right)$ implies MA. (2) CH implies that $\mathrm{MA}_{\mathbb{N}_{1}}$ is false.

Of course if $\mathrm{CH}$ holds, then MA is just $\mathrm{MA}_{\aleph_{0}}$ and of little interest since we can prove the stronger result 1.2. For this reason $\mathrm{MA}$ is often taken to mean MA and $\neg \mathrm{CH}\left(2^{\aleph_{0}}>\aleph_{1}\right)$. In this connection, Solovay and Tennenbaum established the following relative consistency result, which we shall discuss in section 3 .

Theorem 1.10. CON(ZFC $+\mathrm{MA}+\neg \mathrm{CH})$, i.e. the system of axioms of ordinary set theory and MA and $\neg \mathrm{CH}$ is consistent

In other words, if no contradiction can be deduced from ZFC (the axioms of ordinary set theory), then none can be deduced from $\mathrm{ZFC}+\mathrm{MA}+\rightarrow \mathrm{CH}$. We'll often use the equivalent semantic 
formulation: there is a set-theoretic universe (a model of $\mathrm{ZFC}$ ) in which $2^{\aleph_{0}}>\aleph_{1}$ and MA holds. It follows immediately from 1.9 (2) and 1.10 that $\mathrm{MA}_{\aleph_{1}}$ is independent of ordinary set theory, i.e. $\mathrm{MA}_{N_{3}}$ can neither be proved nor refuted from $\mathrm{ZFC}$.

We finish the proofs for this section by showing that $\mathrm{MA}_{\kappa}$ is equivalent to the seemingly weaker axiom $\mathrm{MA}_{\kappa}^{-}$: if $\mathbb{P}$ is a c.c.c. forcing of cardinality at most $\kappa, \mathcal{D}$ is a family of dense sets in $\mathbb{P}$ and $\mathcal{D}$ has cardinality at most $\kappa$, then there is a $\mathcal{D}$-generic filter $G$ in $P$.

Proposition 1.11. $\mathrm{MA}_{\kappa}^{-}$implies $\mathrm{MA}_{\kappa}$.

Proof: Given a family $\mathcal{D}$ of dense sets in an arbitrary forcing $\mathbb{P}$ we find a suitable subforcing $\mathbf{Q}$ of cardinality at most $\kappa$ as follows. Let $c$ be a (partial) function from $P \times P$ to $P$ defined thus: if $p$ and $q$ are compatible, $c(p, q)$ is a common upper bound (otherwise $c(p, q)$ is not defined). For each $D \in \mathcal{D}$, let $c_{D}: P \rightarrow D$ be defined by $c_{D}(p) \in D, p \leq c_{D}(p)$. Now let $Q$ be a non-empty subset of $P$ of cardinality at most $\kappa$ closed under the functions $c$ and $c_{D}$ for $D \in \mathcal{D}$. Easily $\mathrm{Q}=(Q, \leq \uparrow Q)$ is a c.c.c. forcing of cardinality at most $\kappa$, and for $D \in \mathcal{D}, Q \cap D$ is dense in $Q$. So by $\mathrm{MA}^{-}$, there is a filter $H$ in $Q$ intersecting every $Q \cap D$. The filter $G=\{p \in P:(\exists q \in H)(p \leq q)\}$ is now $\mathcal{D}$-generic in $\mathbf{P}$

And to make explicit the connection between the internal forcing axioms of this section and the Baire category theorem, we should point out that 1.3 implies 1.2 and $M A_{\kappa}$ is equivalent to the topological hypothesis: if $X$ is a c.c.c. compact Hausdorff space, then the intersection of at most $\kappa$ dense open subsets of $X$ is nonempty. (Remember that $X$ is c.c.c. means that every collection of pairwise disjoint non-empty sets is countable.)

\section{Section 2: Applications of MA}

In this section we prove some easy independence results (Lusin sets, $Q$-sets) and mention some further applications of MA. Our first aim is to study the effect of MA on the real numbers: what kinds of subsets does $\mathrm{R}$ have?
Recall some Baire catëgory terminology: a subset $N$ of a space $X$ is nowhere dense iff $X \backslash \mathrm{Cl}(N)$ is a dense open set (equivalently, $\operatorname{Int}(\mathrm{Cl}(N))=\emptyset)$; a subset $F$ of $X$ is of first category iff $F$ is a countable union of nowhere dense sets in $X$.

Theorem 2.1. Assume MA. Suppose $X$ is a second countable space. If $\mathcal{F}$ is a family of nowhere dense sets and $\mathcal{F}$ has cardinality $\kappa<2^{\aleph_{0}}$, then $\bigcup \mathcal{F}$ is of first category. For example, MA implies that every set of reals of cardinality less than $2^{\aleph_{0}}$ is of first category, and the category ideal on $\mathbf{R}$ is complete: the union of fewer than $2^{N_{0}}$ first category subsets of $\mathbb{R}$ is of first category.

To prove 2.1 we need a useful combinatorial lemma about $P(\mathrm{~N})$.

Lemma 2.2. Assume $\mathrm{MA}_{\kappa}$. Suppose that $\mathcal{A}$ and $B$ are famil ies of subsets of $\mathrm{N}, \mathcal{A}$ and $B$ have cardinality at most $\kappa$, and if $A_{1}, \ldots, A_{n} \in \mathcal{A}, B \in \mathcal{B}$, then $B \backslash\left(A_{1} \cup \ldots A_{n}\right)$ is infinite. Then there exists $C \subset \mathbb{N}$ such that $C \cap A$ is finite and $C \cap B$ is infinite for all $A \in \mathcal{A}, B \in \mathcal{B}$.

Proof: Write $\mathcal{A}=\left\{A_{i}: i \in I\right\}, \mathcal{B}=\left\{B_{i}: i \in I\right\}$ where $I$ has cardinality $\kappa$ (allowing repetitions if necessary). Define $P$ to be the following set: $\{(h, a): h$ is a finite subset of $I$ and $a$ is a finite subset of $\mathrm{N}\}$; say $(h, a) \leq(k, b)$ iff $h \subset k, a \subset b$ and $(b \backslash a) \cap\left(\bigcup_{i \in h} A_{i}\right)=\emptyset$. It is straightforward to check that $\mathbb{P}=(P, \leq)$ is a forcing. To see that $\mathbf{P}$ is c.c.c., note that $(h, a)$ and $(k, a)$ are compatible for any $h$ and $k$, so if $W \subset P$ is an antichain in $\mathbf{P}$, then $W$ is countable (since there are only countably many possibilities for the second components of elements of $W$ ). It's easy to check that the sets $D_{i}=\{(h, a): i \in h\}$ and $E_{i, n}=$ $\left\{(h, a):\left|a \cap B_{i}\right|>n\right\}$ are dense in $\mathbf{P}$.

Now apply $\mathrm{MA}_{\text {. }}$ to get a filter $G$ intersecting each member of the family $\mathcal{L}=\left\{D_{i}, E_{i, n}: i \in I, n \in \mathbb{N}\right\}(\mathcal{L}$ has cardinality at most $\kappa)$. We'll complete the proof by showing that $C=\bigcup\{a$ : $(\exists h)[(h, a) \in G]\}$ is as required. Fix $i \in I$. Since $G \cap E_{i, n} \neq \emptyset$, it follows that $\left|C \cap B_{i}\right|>n$ for each $n \in \mathbb{N}$ and so $C \cap B_{i}$ is infinite. Also $G \cap D_{i} \neq \emptyset$, so take $(h, a) \in G \cap D_{i}$ and note that 
$C \cap A_{i} \subseteq a$ (if $(k, b) \in G$, then $(b \backslash a) \cap A_{i} \neq \emptyset$ since $(h, a)$ and $(k, b)$ are compatible); so $C \cap A_{i}$ is finite.

Lemma 2.2 for countable collections $\mathcal{A}$ and $B$ is a simple exercise (in ZFC) which does not require any diagonalization. Let's go back now to the proof of Theorem 2.1.

Well, $X$ is second countable, so one can choose a listing $\left\{U_{n}\right.$ : $n \in \mathbb{N}\}$ of a countable basis for the topology on $X$ in which each non-empty basic open set is listed infinitely many times. Let $B_{n}=$ $\left\{m \in \mathbb{N}: U_{m} \subseteq U_{n}\right\} ;$ for $F \in \mathcal{F}$, let $A_{F}=\left\{m \in \mathbb{N}: U_{m} \cap F \neq \emptyset\right\}$ take $\mathcal{A}=\left\{A_{F}: F \in \mathcal{F}\right\}, \mathcal{B}=\left\{B_{n}: n \in \mathbb{N}\right\}$. To see that $\mathcal{A}$ and $B$ satisfy the hypotheses in 2.2 for $\kappa=\max \left(|\mathcal{F}|, \aleph_{0}\right)$, remember that a finite union of nowhere dense sets is nowhere dense and that every basic open set is listed infinitely many times. Apply $\mathrm{MA}_{\kappa}$ to find $C$ as in Lemma 2.2. Let $R_{n}=\bigcup\left\{U_{m}: m \in C\right.$ and $m \geq n\} . R_{n}$ is a dense open subset of $X$ : given $U_{k}$, choose $m \in C \cap B_{k}, m \geq n$; so $U_{m} \subseteq U_{k}$ and $U_{m} \subseteq R_{n}$. Finally let $M_{n}$ be the closed nowhere dense set $X \backslash R_{n}$. It'll suffice to show that $\bigcup \mathcal{F} \subseteq \bigcup_{n \in \mathbb{N}} M_{n}$. For $F \in \mathcal{F}, C \cap A_{F}$ is finite; pick $n \in C \backslash A_{F}, n>\max \left(C \cap A_{F}\right)$, then for every $m \in C, m \geq n$ gives $U_{m} \cap F=\emptyset$, so $F \subseteq \bigcap\left\{X \backslash U_{m}: m \in C, m \geq n\right\}=M_{n}$

In passing, we note that a similar result holds replacing sets of first category by sets of Lebesgue measure zero.

From 1.3 and 2.1 we obtain an independence result. Let $C\left(\aleph_{1}\right)$ abbreviate the assertion: if $A \subseteq \mathbb{R}$ has cardinality $\aleph_{1}$ then $A$ is of first category. We conclude that $C\left(\aleph_{1}\right)$ is independent of ordinary set theory: if CH holds, then $C\left(\aleph_{1}\right)$ is false $(\mathbb{R}$ is a counterexample (by 1.3)); if $\mathrm{MA}+\neg \mathrm{CH}$ holds, then $C\left(\aleph_{1}\right)$ is true (by 2.1).

Before going on to Lusin sets, we need to count the subsets of $\mathbb{R}$.

Proposition 2.3. The following collections have cardinality exactly $2^{\mathrm{K}_{0}}$ : (1) the open sets of reals; (2) the closed sets;

(3) the closed nowhere dense sets.

Proof: Ad (1): Every open set can be expressed as a countable union of open intervals with rational endpoints. There are count- ably many such intervals, so there are at most $\aleph_{0}^{\aleph_{0}}=2^{\aleph_{0}}$ possible choices for open sets of reals. Easily there are at least $2^{\aleph_{0}}$ open sets.

Part (2) follows from (1), closed sets of reals being exactly the complements of open sets; part (3) is immediate from (2).

Lusin sets are a little more obscure than uncountable sets of first category:

Definition 2.4. A subset $K$ of $\mathbf{R}$ is called a Lusin set iff

(i) $K$ is uncountable and

(ii) whenever $F \subseteq \mathbb{R}$ is of first category, then $K \cap F$ is countable.

Lusin sets (discovered of course by Mahlo) are rather unusual: with regard to category, they are not small (no uncountable subset of $K$ is of first category); with regard to Lebesgue measure, they are very small indeed (recall that for every positive $\epsilon$ there is a closed nowhere dense set $N$ such that $\mathbb{R} \backslash N$ has measure less than $\epsilon)$. But are there any Lusin sets? Well, it depends.

Theorem 2.5. (1) $\mathrm{CH}$ implies that there is a Lusin set.

(2) $\mathrm{MA}+\neg \mathrm{CH}$ implies that there are no Lusin sets.

Proof: Ad (1): By 2.3 and $\mathrm{CH}$ we can list all the closed nowhere dense sets in a list $\left\{N_{\alpha}: \alpha<\aleph_{1}\right\}$. Define $K=\left\{r_{\alpha}: \alpha<\aleph_{1}\right\}$ by transfinite induction on $\alpha<\aleph_{1}$. Given $\left\{r_{\beta}: \beta<\alpha\right\}$ note that $M_{\alpha}=\bigcup\left\{N_{\beta}: \beta<\alpha\right\} \cup\left\{r_{\beta}: \beta<\alpha\right\}$ is of first category (since $\alpha$ is a countable ordinal), so by 1.3 one can find $r_{\alpha} \in \mathbb{R} \backslash M_{\alpha}$. By construction, $K$ is a Lusin set: if $F$ is of first category, then for some $\alpha<\aleph_{1}, F \subseteq M_{\alpha}$ and so $K \cap F \subseteq\left\{r_{\beta}: \beta<\alpha\right\}$.

Ad (2): Supposing contrariwise that $K$ is Lusin let $F \subseteq K$ be a subset of cardinality $\aleph_{1}$. By $2.1, F$ is of first category, being the union of its singleton sets - in contradiction to 2.4 (ii). So $K$ doesn't exist.

The $Q$-sets which we define next occur naturally in the study of Moore spaces. We'll explain why after the definition and some basic facts.

Definition 2.6. A set $A \subseteq \mathbb{R}$ is a $Q$-set iff every subset of $A$ is a relative $F_{\sigma}$ (i.e. a countable union of closed sets in the subspace 
topology on $A$ ). For example, every countable set is a $Q$-set. Are there any uncountable $Q$-sets?

Proposition 2.7. (1) If $2^{\aleph_{0}}<2^{\kappa}$, then there are no Q-sets of cardinality $\kappa$.

(2) If $A$ is a $Q$-set, then $A$ has cardinality less than $2^{\aleph_{0}}$. In particular, $\mathrm{CH}$ implies that every $Q$-set is countable.

Proof: Part (2) is a consequence of (1), noting that $\lambda<2^{\lambda}$ and taking $\lambda=2^{\aleph_{0}}$. As regards (1), suppose that $B$ has cardinality $\kappa$. By 2.3 there are at most $2^{\aleph_{0}}$ relatively closed subsets of $B$, so there are at most $\left(2^{\aleph_{0}}\right)^{\aleph_{0}}=2^{\aleph_{0}}$ relative $F_{\sigma}$ 's of $B$. However, $|P(B)|=2^{|B|}=2^{\kappa}>2^{\aleph_{0}}$, so some subset of $B$ is not a relative $F_{\sigma}$, i.e. $B$ is not a $Q$-set.

Theorem 2.8. Assume MA. (1) Every set of reals of cardinality less than $2^{\aleph_{0}}$ is a $Q$-set.

(2) For $\aleph_{0}<\kappa<2^{\aleph_{0}}, 2^{\kappa}=2^{\aleph_{0}}$. (3) $2^{\aleph_{0}}$ is a regular cardinal.

Proof: Part (1) is similar to 2.1 and we give just a sketch. Sup pose $X \subseteq A \subseteq \mathbb{R}$ and $A$ has cardinality $\kappa<2^{\kappa_{o}}$. We show $X$ is a relative $F_{\sigma}$. WLOG $X$ is a non-empty proper subset of $A$. Choose a countable open basis $\left\{V_{n}: n \in \mathbb{N}\right\}$ for $\mathbb{R}$ such that no two different reals belong to the intersection of infinitely many $V_{n}$. Let $O_{x}=\left\{n \in \mathbb{N}: x \in V_{n}\right\}$ and note that $\mathcal{A}=\left\{O_{x}: x \in X\right\}$ and $\mathcal{B}=\left\{O_{x}: x \in A \backslash X\right\}$ satisfy the hypotheses of 2.2. Using $C$ from 2.2 , the open sets $G_{n}=\bigcup\left\{V_{k}: k \in C\right.$ and $k \geq n\}$, the closed sets $F_{n}=\mathbb{R} \backslash G_{n}$, one verifies that $X \subseteq \bigcup\left\{F_{n}: n \in \mathbb{N}\right\}, A \backslash X \subseteq \bigcup\left\{G_{n}: n \in \mathbb{N}\right\}$ and so $X$ is a relative $F_{\sigma}$.

Ad (2): Let $B \subseteq \mathbb{R}$ have infinite cardinality $\kappa<2^{\aleph_{0}}$. By part (1), $B$ is a $Q$-set, hence by $2.7(1), 2^{\aleph_{0}}=2^{\kappa}$. Ad (3): Since $\mathbb{R}$ has cardinality $2^{\aleph_{0}}$, we work with $\mathbb{R}$. If $\mathbb{R}=\bigcup\left\{A_{i}: i<\lambda\right\}$ where $\left|A_{i}\right|<2^{\aleph_{0}}$, then by 2.1 each $A_{i}$ is of first category and so by 2.1 again $\lambda \geq 2^{\aleph_{0}}$. (The reader familiar with Koenig's Lemma will deduce part (3) immediately from part (2).)

Thus MA $+\neg \mathrm{CH}$ implies that there are uncountable $Q$-sets. Taken in conjunction with 2.7 (2) this means that the existence of an uncountable $Q$-set is independent of $\mathrm{ZFC}$.
From 2.8 (3) it also follows that $\neg \mathrm{CH}$ does not imply MA, since there are set-theoretic universes in which $2^{\aleph_{0}}$ is not a regular cardinal.

Uncountable $Q$-sets are related to the Normal Moore Space Conjecture (NMSC) which states that all normal Moore spaces are metrizable. A space is normal iff for all disjoint closed sets $A$ and $B$ there are disjoint open sets $U$ and $V, A \subseteq U, B \subseteq V$. A Moore space is a regular space $X$ with a sequence of open covers $\left\{\mathcal{G}_{n}: n \in \mathbb{N}\right\}$ such that for each $x \in X$ and open $U$ with $x \in U$, there is $n \in \mathbb{N}$ such that $\bigcup\left\{G \in \mathcal{G}_{n}: x \in G\right\} \subseteq U$. Examples of normal non-metrizable Moore spaces can be obtained in the following way.

Example 2.9. For this we take an uncountable set $B \subset \mathbb{R}$. Let $M(B)$ be the set $B \cup\left\{(x, y) \in \mathbb{R}^{2}: y>0\right\}$; the neighbourhoods of $b \in B$ are the bubbles at $b$, i.e. $\{b\} \cup \operatorname{Int}(D)$ where $D$ runs ove the discs in the upper half-plane tangent to the $x$-axis at $(b, 0)$; the neighbourhoods of $(x, y)$ are the usual Euclidean ones. $M(B)$ is called the Moore space derived from $B$ and is a separable nonmetrizable Moore space. It turns out that $M(B)$ is normal iff $B$ is a $Q$-set. It is also known that the existence of an uncountable $Q$-set is equivalent to the existence of a separable normal nonmetrizable Moore space. So MA $+\neg \mathrm{CH}$ implies that NMSC is false, even in the separable case. Of course this leaves open the question whether the falsity of NMSC follows just from ordinary set theory. The resolution of this issue is a little different from the independence results we've considered so far. It involves so-called large cardinal axioms, axioms which roughly speaking assert the existence of cardinals so large that they cannot be shown to exist on the basis of ordinary set theory. We state the result, omitting the technical definitions and details:

Theorem 2.10. (1) If NMSC holds, then there is an inner model of ZFC with a measurable cardinal. (2) The Product Measure Extension Axiom (PMEA) implies NMSC. (3) If $\mathrm{ZFC}+$ "there is a strongly compact cardinal" is consistent, then $\mathrm{ZFC}+\mathrm{PMEA}$ is consistent, and so ZFC + NMSC is consistent.

Before leaving metrizability questions, let us mention an ap- 
plication of MA in the study of manifolds. Taking a manifold to be a connected regular Hausdorff space $M$ for which there is a positive integer $n$ such that each point of $M$ has a neighbourhood homeomorphic to $\mathbb{R}^{n}$, one can prove:

Theorem 2.11. (1) Assume MA $+\neg \mathrm{CH}$. Then every perfectly normal manifold is metrizable.

(2) There is a set-theoretic universe $L$ in which there exists a perfectly normal non-metrizable manifold.

Thus again the answer to a query of Alexandroff is independent of $\mathrm{ZFC}$.

Our next application concerns the uniqueness of the real line $(\mathbb{R}, \leq)$. Suslin's Hypothesis claims that there are no Suslin trees. Recall that a Suslin tree is an uncountable c.c.c. partial order $T=(T, \leq)$ satisfying: (a) $(\forall t \in T) \operatorname{Pred}(t)=\{s \in T: s<t\}$ is a chain which is well-ordered, i.e. every non-empty subset of Pred $(t)$ has a <-least element; (b) $T$ has no uncountable chains; (c) $(\forall t \in T) \operatorname{Suc}(t)=\{s \in T: t<s\}$ is uncountable. The study of Suslin's Hypothesis led to the discovery of Martin's Axiom.

Theorem 2.12. $\mathrm{MA}_{\aleph_{\mathrm{I}}}$ implies SH: there are no Suslin trees.

Proof: Suppose for a contradiction that $T$ is a Suslin tree. By (a) and (b), Pred $(t)$ is order-isomorphic to a countable ordinal $h(t)$, the height of $t$ in $T$, so (c) implies that the set $D_{\alpha}=\{t \in$ $T: h(t) \geq \alpha$ is dense in $T$ for each ordinal $\alpha<\aleph_{1}$. Apply MA $_{\aleph_{1}}$ to find a filter $G$ in $T$ intersecting each $D_{\alpha}$ non-trivially. Now $G$ is an uncountable chain in $T$, contradicting (b).

It is consistent with ordinary set theory to assume that SH is false. For example, in $L$ (the smallest transitive set-theoretic universe containing all the ordinals) there is a Suslin tree.

Most mathematics students learn (in a possibly different terminology) that if $(S, \leq)$ is a separable, uncountable, unbounded, Dedekind-complete, dense linear order, then $(S, \leq)$ is order isomorphic to the real line $(\mathbf{R}, \leq$ ) (just recall the well-known backand-forth argument of Cantor characterizing the rational line $(\mathrm{Q}$, $\leq)$ ). Suslin's Hypothesis is equivalent to the assertion that separ- ability can be replaced by the condition that every collection of pairwise disjoint open intervals in the linear order is countable.

Finally we turn to two famous applications of $\mathrm{MA}$ in algebra and analysis. We say that an infinite abelian group $A$ is a free group iff $A$ has a linearly independent set of generators; we say that $A$ is a $W$-group iff for every surjective homomorphism $\pi: B \rightarrow A$ with kernel $\mathbb{Z}$ there is a homomorphism $\phi: A \rightarrow B$ such that $\pi(\phi(a))=a$ for all $a \in A$ (in other words $\operatorname{Ext}(A, \mathbb{Z})=0$ ). For example, every free group is a $W$-group. Whitehead asked: is every $W$-group free? Shelah showed that $M A_{\aleph_{1}}$ implies the existence of a non-free $W$-group. He was also able to prove that in $L$ every $W$-group is free. So the Whitehead problem is independent of $\mathrm{ZFC}$. It is remarkable that the concepts involved in his research yield, via trees, considerable information on NMSC.

Let's conclude this section with an automatic continuity problem in analysis. Recall that $C[0,1]$ is the commutative Banach algebra of continuous functions on the closed unit interval. Kaplansky's question asks: is every homomorphism from $C[0,1]$ into a commutative Banach algebra continuous? Assuming MA ${ }_{N_{1}}$ one can build a set-theoretic universe in which the answer is positive. On the other hand, in $L$ the answer is no, so again Kaplansky's question is independent of $\mathrm{ZFC}$.

Section 3: Proper forcing and the Proper Forcing Axiom In section 1 we introduced the countable chain condition in a rather ad hoc manner, essentially to obviate the counterexamples arising in 1.4. That end might be achieved by other means. For example, regarding $\mathrm{MA}_{\aleph_{1}}$, the first independent instance of MA, it is natural to inquire whether there is a weak property of forcings, implied by the c.c.c., for which there is a consistent internal forcing axiom of the form: if $\mathbf{P}$ has the property, $\mathcal{D}$ is a family of dense sets and $\mathcal{D}$ has cardinality at most $\aleph_{1}$, then there is a $\mathcal{D}$-generic filter $G$ in P. How should one look for such a property? Well, in this context, the important point about $\mathrm{MA}$ and $\mathrm{MA}_{\mathrm{N}_{1}}$ is the relative consistency theorem 1.10 . One could start by analysing the proof of 1.10. This is one of the tasks in Shelah's monograph $[18$, p.200]. We review briefly the ideas to motivate the concept 
of proper forcing and the Proper Forcing Axiom PFA.

The basic strategy in the relative consistency proof of MA $+\neg \mathrm{CH}$ is to start from a set-theoretic universe $V_{0}$ (in which $\mathrm{CH}$ holds) and to build a bigger set-theoretic universe $V_{\star}$ in which MA $+\neg \mathrm{CH}$ holds. We build $V_{\star}$ in stages and each stage is called an iteration. The construction of a stage goes roughly as follows. Given a set-theoretic universe $V$, a forcing $\mathbf{P} \in V$ and a filter $G$ in $\mathbb{P}$ which is generic over $V$ (i.e. $G$ is $\mathcal{D}$-generic where $\mathcal{D}$ is the set $\{D \in V: D$ is dense in $\mathbf{P}\}$ ), then there is a smallest set-theoretic universe $V[G]$ such that $V \subseteq V[G]$ and $G \in V[G]$. Except in trivial cases, $G \notin V$, so $V[G]$ is a bigger universe than $V$. For example, if $\mathbf{P}$ is the Cohen forcing of 1.1 and $G$ is generic over $V$ then the Cohen real $\bigcup G$ is a real belonging to $V[G]$ but not to $V$. Now extending $V$ to $V[G]$ is not without potential danger. For example, suppose that $\aleph_{1}^{V}$ is the first uncountable cardinal in $V$; if $V[G]$ should chance to contain a function from $\mathrm{N}$ onto $\aleph V$, then $\aleph_{1}^{V}$ is a countable set in $V[G]$, so that $\aleph_{1}^{V[G]}$, the first uncountable cardinal in $V[G]$, is greater than $\aleph_{1}^{V}$. In this situation, we say that $\mathbf{P}$ collapses $\aleph_{1}$. If on the other hand $\aleph_{1}^{V}$ is $\aleph_{1}^{V[G]}$, then we say that $\mathbf{P}$ preserves $\aleph_{1}$. The proofs that $\mathrm{MA}_{\aleph_{1}}$ and $\mathrm{MA}+\neg \mathrm{CH}$ are consistent rely on three principal facts: (1) If $\mathbf{P}$ is c.c.c., then $P$ preserves $\aleph_{1} ;(2)$ there is an iterative operation under which the class of c.c.c. forcings is closed; (3) $\mathrm{MA}_{\kappa}$ is equivalent to $\mathrm{MA}_{\kappa}^{-}$. (We actually verified (3) in 1.11.)

From this very brief sketch we learn that each property of forcings for which analogues of facts (1), (2) and (3) obtain, will give rise to a consistent internal forcing axiom. One of the most interesting and powerful among these properties is properness. There are several equivalent definitions of properness. We give one which allows an easy proof that proper forcings preserve $\aleph_{1}$.

Definition 3.1. (1) Let $A$ be an uncountable set. We use $[A]^{\aleph_{0}}$ to denote the collection of countable subsets of $A$. A subset $C$ of $[A]^{\aleph_{0}}$ is a club (closed unbounded set) iff (i) every element of $[A]^{\aleph_{0}}$ is contained in an element of $C$ and (ii) for every increasing sequence $x_{0} \subseteq x_{1} \subseteq \ldots \subseteq x_{n} \subseteq \ldots, x_{n} \in C$, the union $\bigcup_{n \in \mathrm{N}} x_{n} \in C$.
(2) A subset $S$ of $[A]^{\aleph_{0}}$ is stationary in $[A]^{\aleph_{0}}$ iff $S \cap C \neq \emptyset$ for every club $C$.

(3) For a set-theoretic universe $M$ and a set $A \in M$, we write $\left([A]^{N_{0}}\right)^{M}$ for the set $\{x \in M:$ in $M, x$ is a countable subset of A\}.

Definition 3.2. A forcing $\mathbb{P} \in V$ is proper iff for every uncountable set $A \in V$, if $S \in V$ is stationary in $\left([A]^{N_{0}}\right)^{V}$, then $S$ is stationary in $\left([A]^{N_{0}}\right)^{V[G]}$ for every filter $G$ in $P$ generic over $V$. Loosely put, proper forcings preserve stationarity.

To exercise these definitions a little, let's prove proper forcings preserve $\aleph_{1}$.

Theorem 3.3. Suppose that $\mathbf{P} \in V$ is proper and $G$ is a generic filter over $V$. If in $V[G]$ the set $a$ is a countable set of ordinals, then in $V$ there is a countable set $b$ of ordinals such that $a \subseteq b$. Thus $\aleph_{1}^{V}=\aleph_{1}^{V[G]}$.

Proof: Since in $V[G] a$ is countable, there is an uncountable cardinal $\lambda$ with $a \in\left([\lambda]^{N_{0}}\right)^{V[G]}$. In $V[G]$,

$$
C=\left\{x \in\left([\lambda]^{\aleph_{0}}\right)^{V[G]}: a \subseteq x\right\}
$$

is a club. But $S=\left([\lambda]^{\aleph_{0}}\right)^{V}$ is stationary in $\left([\lambda]^{\aleph_{0}}\right)^{V}$, hence $S$ is stationary in $\left([\lambda]^{N_{0}}\right)^{V[G]}$ since $\mathbb{P}$ is proper. Therefore $S \cap C \neq \emptyset$. Choose $b \in S \cap C$.

Definition 3.4. The Proper Forcing Axiom PFA is the hypothesis: if $\mathbf{P}$ is a proper forcing, $\mathcal{D}$ is a family of dense sets in $\mathbb{P}$ and $\mathcal{D}$ has cardinality at most $\mathfrak{N}_{1}$, then there is a $\mathcal{D}$-generic filter $G$ in $\mathbf{P}$.

The Proper Forcing Axiom is the analogue of $\mathrm{MA}_{\mathrm{N}_{1}}$ for proper forcings. We finish by noting some basic theorems.

Theorem 3.5. (1) If $\mathbb{P}$ is a c.c.c. forcing, then $\mathbb{P}$ is proper. So PFA implies $\mathrm{MA}_{\aleph_{1}}$. (2) PFA implies $\mathrm{MA}_{\mathbb{N}_{2}}$ is false.

(3) PFA implies $2^{\aleph_{0}}=\aleph_{2}$. So PFA implies MA. 
Theorem 3.6. If ZFC+ "there is a supercompact cardinal" is consistent, then $\mathrm{ZFC}+\mathrm{PFA}$ is consistent.

The large cardinal axiom in 3.6 is used to establish the appropriate version of 1.11 for proper forcings. There are variants of PFA which do not require any large cardinal axioms for their consistency proofs. There are also even stronger axioms (Martin's Maximum MM) which are studied in the literature.

\section{Section 4: Bibliographical notes}

Martin's Axiom is the eponymous subject of the monograph [6, p.200]. Good brief introductions to MA are [19, p.200], [17, p.200], chapter 2 in [11, p.200] and perhaps [22, p.200]

On $Q$-sets, see [14, p.200]. NMSC is covered in [20, p.200] and $[5$, p.200]. The articles $[7$, p.200] and $[16$, p.200] provide good accounts of the impact of logic and recent set theory. The book $[4, \mathrm{p} .200]$ is an excellent text on set-theoretic methods in algebra with many applications of MA and PFA. The lecture notes in [3, p.200] deal with MA in analysis (Kaplansky's conjecture); $[15$ p.200] presents the solution to the Alexandroff problem and is an introduction to non-metrizable manifolds.

Proper forcings and variants appear in [18, p.200]. Applications are in $[1$, p.200], $[2$, p.200], $[9$, p.200] and $[4$, p.200]. A very interesting variant of $\mathrm{PFA}$ which does not require a large cardinal axiom in its consistency proof can be found in [13, p.200].

An extensive account of large cardinal axioms is provided in $[10$, p.200] or in $[8$, p.200]. $[8$, p.200] and $[11$, p.200] cover all the axiomatic set theory which we didn't. Iterations are treated in [1, p.200], [11, p.200] and [9, p.200]. [21, p.200] has the proof of 3.5 (3).

\section{References}

[1] J. Baumgartner, Iterated forcing in Surveys in set theory, Cambridge University Press, 1983, 1-59.
[2] 3. Baumgartner, Applications of the proper forcing axiom in [12, p.200], 1984, 913-959.

[3] H. G. Dales and W. H. Woodin, An introduction to independence for analysts, (London Math. Soc. Lecture Notes 115). Cambridge University Press, 1986.

[4] P. C. Eklof and A. H. Mekler, Almost free modules. North-Holland, 1990.

[5] W. G. Fleissner, The normal Moore space conjecture and large cardinals in [12], 1984, 733-760.

[6] D. H. Fremlin, Consequences of Martin's Axiom, (Cambridge Tracts in Mathematics 84). Cambridge University Press, 1984.

[7] B. Goldsmith, Realizing rings as endomorphism rings - the impact of logic, Bull. Irish Math. Soc. 17 (1986).

[8] T. Jech, Set theory. Academic Press, 1978.

[9] T. Jech, Multiple forcing, (Cambridge Tracts in Mathematics 88). Cambridge University Press, 1986.

[10] A. Kanamori and M. Magidor, The evolution of large cardinal axioms in set theory in Higher set theory. Lecture Notes in Mathematics 669, Springer-Verlag, 1978, 99-275.

[11] K. Kunen, Set theory. North-Holland, 1980.

[12] K. Kunen and J. E. Vaughan editors, Handbook of set-theoretic topology. North-Holland, 1984.

[13] A. H. Mekler, Proper forcing and abelian groups in Abelian group theory. Lecture Notes in Mathematics 1006, Springer-Verlag, 1983, 285-303.

[14] A. W. Miller, Special subsets of the real line in [12], 1984, 201-233.

[15] P. Nyikos, The theory of non-metrizable manifolds in [12], 1984, 533684.

[16] J. Roitman, The uses of set theory, Math. Intelligencer 14(1) (1992), 63-69.

[17] M. E. Rudin, Martin's Axiom in Handbook of mathematical logic North-Holland, 1977, 491-501.

[18] S. Shelah, Proper forcing. Springer-Verlag, Lecture Notes in Mathematics 940, 1982 (A revised augmented edition is due out soon).

[19] J. Shoenfield, Martin's Axiom, Amer. Math. Monthly 82(6) (1975) $610-617$

[20] F. D. Tall, Normality versus collectionwise normality in [12], 1984. 
[21] B. Velicković, Forcing axioms and stationary sets, Advances in Math. (to appear).

[22] W. Weiss, Versions of Martin's Axiom in [12], 1984, 827-886.

\section{Eoin Coleman}

Department of Mathematics

College of Technology

Kevin Street

Dublin

\section{EXPLICIT RELATIONSHIPS BETWEEN ROUTH-HURWTTZ AND SCHUR-COHN TYPES OF STABILTY}

\author{
Ziad Zahreddine
}

Abstract: Given two linear systems of differential equations with real or complex coefficients, and of the same arbitrary dimension. Suppose bor in the Schur-Cohn sense. We directly express the coefficients of each in the Schur-Cohn sense. We directly express the coefficients of each plicit, make it possible to convey any stability criterion of either of the two types to the other.

\section{Introduction}

The concept of stability in differential equations has been defined in many different ways. Among these various definitions are the well-known Routh-Hurwitz and Schur-Cohn types of stability. Given a linear system of differential equations, the classical Routh-Hurwitz problem is that of obtaining necessary and sufficient conditions for all eigenvalues of the system to lie in the left half of the complex plane. The Schur-Cohn problem is that of establishing necessary and sufficient conditions for all eigenvalues to lie within the unit circle. Solutions to these problems have been the subject of intensive research over the last few years [2], $[3],[9],[12]$ and [14].

It is often noticed in the literature that some interesting results about stability, in the Hurwitz sense for example, triggers an

AMS subject classification: primary $34 \mathrm{D}$, secondary $93 \mathrm{D}$.

Key words and phrases: Routh-Hurwitz stability, Schur-Cohn stability, continuous-time systems, discrete-time systems. 